\title{
Justicia Social, Culturas de Paz y Competencias Digitales: Comunicación para una Ciudadanía Crítica Global en la Educación Superior
}

\author{
Social Justice, Cultures of Peace and Digital Competences: \\ Communication for Critical Global Citizenship in Higher \\ Education
}

\author{
Eloísa Nos Aldás * \\ Alessandra Farné \\ Tamer Al-Najjar Trujillo \\ Universitat Jaume I, España
}

\begin{abstract}
Este artículo presenta una revisión de la fundamentación teórica, la implementación y la evaluación de propuestas de aprendizaje crítico basado en valores de paz, igualdad y justicia social global. Para ello, este trabajo aborda el caso de la Comunicación transgresora de Cambio Social en la educación superior por medio de metodologías participativas y cooperativas para la adquisición de competencias comunicativas y digitales orientadas a los Objetivos de Desarrollo Sostenible. Además, se plantean los principales retos educativos en el espacio universitario, teniendo en cuenta el papel que desempeñan las redes sociales digitales en los procesos de aprendizaje y en la construcción de identidades. En concreto, analizamos dos asignaturas de la Universitat Jaume I de Castellón (España): “Comunicación para la igualdad”, del Grado en Publicidad y Relaciones Públicas, y "Comunicación para la paz”, en el Máster Universitario en Estudios de Paz, Conflictos y Desarrollo. Los planteamientos que aquí se recogen están fundamentados en una concepción de la enseñanza superior como una comunidad de aprendizaje para una ciudadanía crítica y activa capaz de localizar las raíces de la violencia cultural para imaginar y promover otras realidades, mediante la creación de nuevos relatos. Basándonos en la tradición de una pedagogía crítica y de una comunicación de cambio social, nos centramos en detectar discursos transformadores exitosos para aprender a construir otras opciones políticas a través de la comunicación.
\end{abstract}

Descriptores: Cambio social; Comunicación; Cultura de paz; Educación superior; Justicia social.

This paper presents a revision of the theoretical grounding, implementation and evaluation of a proposal of critical learning based on values of peace, equality and global social justice. The article addresses the case of transgressive communication of social change in higher education through participatory and cooperative methodologies to acquire communicative and digital competences in the context of the Sustainable Development Goals. In addition, we address the main educational challenges in the university context considering the role played by the digital social media in the learning process and the configuration of identities. Specifically, we analyse two courses of the Universitat Jaume I of Castellón (Spain): "Communication towards equality", in the undergraduate in Advertising and Public Relations, and "Communication for peace", of the Master in International Studies in Peace, Conflict and Development. These proposals are grounded on a conception of higher education

*Contacto: aldas@uji.es

ISSN: 2254-3139

www.rinace.net/riejs/

revistas.uam.es/riejs
Recibido: $\quad 1$ de febrero de 2019

$1^{\text {a }}$ Evaluación: 24 de abril de 2019

$2^{\text {a }}$ Evaluación: 9 de mayo de 2019

Aceptado: $\quad 10$ de mayo de 2019 
as a learning and communicative community as critical and active citizens able to trace the root causes of cultural violence in order to imagine and promote other realities, by creating alternative discourses. We rely on critical pedagogy and transgressive communication of social change to focus on detect successful transformative discourses to learn how to build other political options through communication.

Keywords: Communication; Culture of peace; Higher education; Social change; Social justice.

\section{Introducción}

En 2015, las Naciones Unidas reformularon sus metas a través de los Objetivos de Desarrollo Sostenible (ODS) impulsados a nivel mundial en su Agenda 2030. La UNESCO (2012) puso el énfasis en el rol de una educación crítica para implicar a la ciudadanía en la implementación del desarrollo sostenible, entendido en sus facetas social, económica y medioambiental, así como cultural, por el papel determinante de las personas que lo tenemos que comprender, apoyar y llevar a cabo. De ahí la necesaria colaboración entre todos los actores sociales, a través de un último objetivo (el 17) denominado "Alianzas para lograr los objetivos" (Naciones Unidas, 2015).

En línea con dicho proyecto, este artículo comparte pedagogías y experiencias innovadoras en el entorno universitario que se desprenden de la práctica docente en dos asignaturas desarrolladas en la Universitat Jaume I de Castellón: "Comunicación para la igualdad”, en el Grado de Publicidad y Relaciones Públicas, y "Comunicación para la paz”, en el Máster Universitario en Estudios de Paz, Conflictos y Desarrollo. El objetivo de este trabajo es revisar tanto los enfoques teóricos y pedagógicos como las experiencias de estos cursos vinculados a la formación de una ciudadanía crítica comprometida con valores de paz, igualdad y justicia social global.

De esta manera, siguiendo la mirada propositiva de los Estudios para la Paz (Galtung, 1996), esta investigación presenta alternativas desde el espacio educativo superior que posibiliten la transformación social a través de prácticas vinculadas con la educación para la ciudadanía crítica global, entendida esta como "un factor de transformación" que trabaja con habilidades, valores y actitudes "para poder contribuir a un mundo más inclusivo, justo y pacífico" (UNESCO, 2015, p. 15). Es por ello que las asignaturas aquí recogidas tienen vinculación con los valores de la justicia social, la noviolencia, los derechos humanos y la igualdad (Naciones Unidas, 1999), como un primer paso para la promoción y construcción de Culturas para la Paz (Martínez Guzmán, 2001, 2005) y la consecución de los ODS.

Los 17 ODS se encuentran de forma transversal en el currículum de las dos asignaturas que presentamos. Concretamente, "Comunicación para la igualdad" está estrechamente vinculada con el objetivo (5) "Igualdad de género" y, por estar en el ámbito de los estudios de publicidad, también el (12) "Una producción y consumo responsables". En el caso de "Comunicación para la paz", está muy vinculada al objetivo (16) "Paz, justicia e instituciones sólidas" y al (5) "Igualdad de género". Además, los dos cursos están conectados al (4) "Educación de calidad", al (10) "Reducción de las desigualdades" y al (17) "Alianzas para lograr los objetivos", precisamente por el rol que la ciudadanía comprometida necesita asumir para colaborar en la implementación de todos los ODS.

Por consiguiente, la formación universitaria vinculada a estas asignaturas supone el desarrollo de prácticas pedagógicas transformadoras con el objetivo de estimular el 
pensamiento crítico y activo del estudiantado, resaltando la importancia de los efectos sociales, culturales y políticos de la comunicación (Ramonet, 1998). De lo que se trata, pues, es de crear espacios de capacitación del estudiantado, en los que se combina práctica y teoría, para destacar la importancia de la comunicación en la transformación de las desigualdades, las injusticias sociales y los conflictos, que pasa por entender sus causas profundas y su interconexión a escala global. Estos procesos de aprendizaje permitirán conformar ciudadanías más solidarias, críticas y participativas: "adoptar un comportamiento ético ante las cosas que pasan ante nuestras miradas, en nuestra proximidad más cercana, como individuos y seres sociales, y, también, en esa aldea global en la que todos vivimos" (Tuvilla Rayo, 2004, p. 405).

Por un lado, los programas pedagógicos que aquí se presentan pretenden sentar las bases para desarrollar un modelo educativo emancipador, a través del cual poner en marcha prácticas comunicativas "transgresoras" (Hooks, 1994; Lagarde y de los Ríos, 2005) con el objetivo de transformar los marcos hegemónicos y desarrollar "prácticas pedagógicas comprometidas con crear un nuevo lenguaje, superar las fronteras disciplinarias, descentrar la autoridad, y reescribir los límites institucionales y discursivos en los que la política se convierte en una condición para reafirmar la relación entre capacidad, poder y lucha" (Giroux y McLaren, 1993; citado en Hooks, 1994, p. 129)․․ Por otro, suponen la implementación de espacios de aprendizaje colectivos, recíprocos y reflexivos donde el estudiantado aprenda a "plantearse problemas, a analizarlos; a buscar soluciones alternativas y compararlas, a contrastarlas con las opiniones de los otros y poder valorar cuáles son las mejores" (Oxfam, 2007, p. 276).

Es por ello que la puesta en marcha de ambos programas docentes, que se fundamentan en las siguientes páginas, pasa por convertir los espacios de enseñanza-aprendizaje, dentro y fuera de las aulas, en una comunidad de comunicación capaz de imaginar otras realidades a lo largo de su proceso formativo como ciudadanía activa y creadora de acciones transformadoras como profesionales de la comunicación.

\section{Pedagogía crítica y comunicación transgresora del cambio social}

El desarrollo de las metodologías docentes que aquí se presentan, se basa en una pedagogía crítica que supone enfocar la docencia en comunicación en generar narrativas alternativas (Nos Aldás, 2019). Es decir, no solo basta con criticar las malas prácticas, sino que, además, es necesario detectar discursos transformadores exitosos para aprender a construir otras opciones políticas a través de la implementación de acciones comunicativas (Duncombe, 2007).

De esta manera, la base teórica y metodológica en la que se asientan estas propuestas pedagógicas tienen un enfoque interdisciplinar y comparativo, además de una estrecha vinculación con la Comunicación para el Desarrollo y el Cambio Social (CDSC), en relación con la Educación para el Desarrollo (EPD) y su tránsito hacia el concepto de Educación para la ciudadanía global (EPCG) (Erro, 2010; Mesa, 2010; Torres, 2017). Es decir, este último concepto se entiende como una herramienta que permite la comprensión crítica de

${ }_{1}^{1}$ Traducción propia del original en inglés. 
la realidad donde se conecta lo local y lo global: “Apuesta por una enseñanza orientada al compromiso y a la acción, que aliente al estudiantado a responsabilizarse, a tomar decisiones y a mejorar su entorno” (Bugallo Rodríguez y Naya Riveiro, 2018, p. 141). Por lo tanto, se parte de la idea de que una lectura reflexiva y crítica de las distintas realidades supone un primer paso para contribuir a una "reconstrucción cultural y social” (Oxfam, 2007, p. 118).

Así pues, la implementación de este tipo de educación comienza a desarrollarse a partir de las aportaciones realizadas desde las Organizaciones no Gubernamentales para el Desarrollo (Bourn, 2018), haciendo énfasis en el importante papel que desempeña la EPD a nivel global y el tipo de eficacia educativa necesaria para su comunicación (Pinazo y Nos Aldás, 2015). Específicamente, destacamos las propuestas internacionales desarrolladas dentro del campo de la Comunicación para el Cambio Social que fomentan la comprensión de los problemas a través de su dimensión social y global. Por ejemplo, a través del proyecto DevReporter (2016), que ha elaborado un Vademécum para una información internacional responsable donde se explica la necesidad de: "Poner en evidencia la relación entre las causas de las problemáticas y sus impactos en diferentes países del Norte y del Sur” (p. 2). Por otro lado, algunos medios periodísticos, como es el caso de United Explanations (2018), también se sitúan en esta línea de actuación al entender la información internacional con un enfoque de derechos humanos (Arévalo Salinas, Vilar Sastre y Al-Najjar, 2018, p. 21).

Para lograr representaciones complejas, completas, representativas, movilizadoras y transgresoras, se recogen los valores inclusivos, universales y emancipadores desarrollados por Mesa, Alonso Cano y Couceiro (2013) vinculados a las historias de vida de 1325 mujeres pacifistas como criterios para una comunicación de eficacia cultural en el marco de la comunicación transgresora y de cambio social (figura 1).

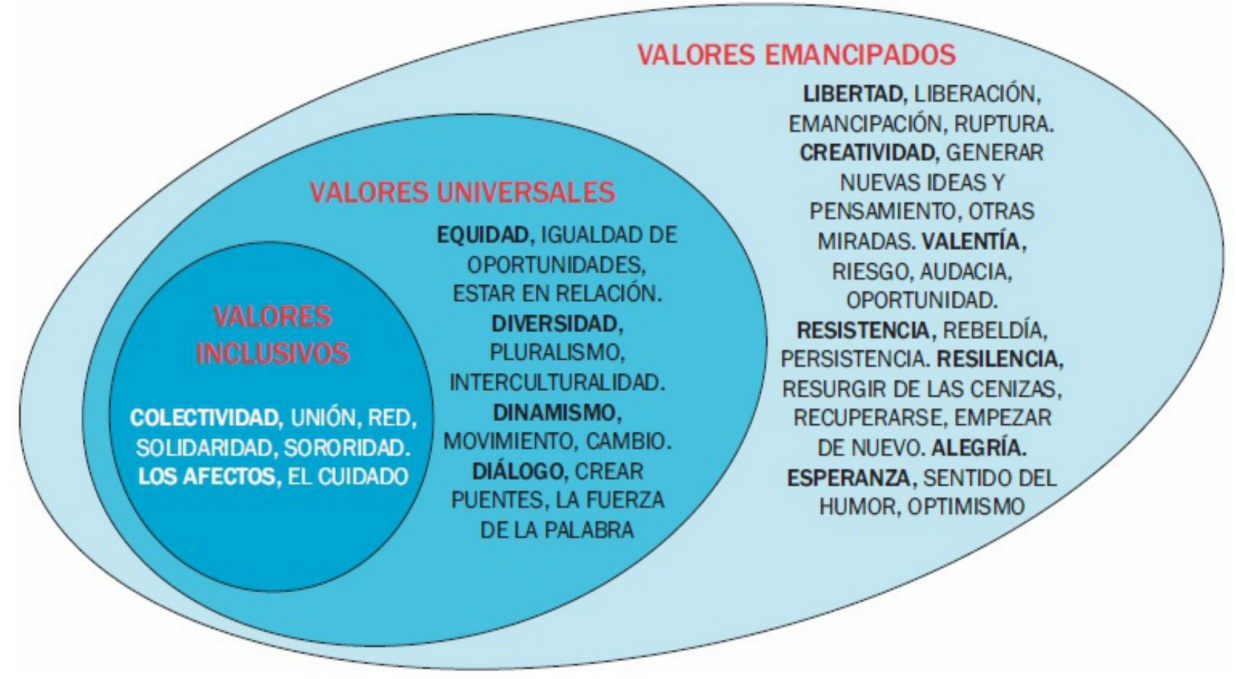

Figura 1. Comunicación y valores transgresores

Fuente: Recuperado de Mesa, Alonso Cano y Couceiro (2013, p. 46).

De este modo, la CDCS requiere la creación de otras estrategias comunicativas ligadas a las capacidades, la justicia social y la libertad (Tufte, 2015, 2017), sin dejar de lado los estudios sobre las epistemologías del Sur y la descolonización (Santos, 2012; Sen, 1999). Para ello es imprescindible el replanteamiento de los modelos económico-financieros, 
estrechamente vinculados con el sistema mediático y publicitario hegemónico, para fomentar una economía crítica, social, solidaria, sostenible y eco-feminista (Herrero, 2013; Laville y Jané, 2009; Mies y Shiva 1993).

La comunicación pasaría a entenderse como un proceso educativo y transformador que posibilita la participación y el empoderamiento de diferentes agentes sociales. La CDCS se sustenta en "Un modelo de comunicación que supere los principios etnocéntricos, androcéntricos, patriarcales y economicistas/desarrollistas de occidente, y que sea capaz de conseguir la implicación, el compromiso y la movilización de los actores sociales para transformar violencias e injusticias a través de medios pacíficos" (López Ferrández, 2016, p. 96).

Para ello, este enfoque pedagógico se asienta en el concepto de interseccionalidad (Crenshaw, 1991), siendo uno de los temas abordados dentro de las asignaturas y que impregnan sus enfoques y casos de estudio. Se reflexiona sobre la marginalización y exclusión como procesos complejos en los que entran en juego múltiples variables: estructurales, políticas y representativas, vinculadas con el género, la etnia, el origen geográfico, la orientación sexual, las clases o la edad, entre otras.

Las bases de este modelo comunicativo se plantean desde la importancia de los discursos para construir e imaginar escenarios sociales y culturales, siguiendo la ética del discurso sobre la performatividad del lenguaje (Austin, 1976). Para el estudio de los efectos socioculturales del lenguaje y el análisis del papel que desempeña la comunicación en dichos procesos es necesario tener en cuenta el bagaje teórico de los estudios culturales, las propuestas postcoloniales y los estudios de género y queer que entienden el discurso como una representación cultural y su influencia en la construcción de identidades (Hall, 1997). Dicha concepción de la comunicación posibilita el diseño de programas de aprendizaje en el que el estudiantado asuma y sea consciente de la responsabilidad de sus acciones y discursos dentro de su entorno, pero, también, a nivel internacional. Desde un punto de vista pedagógico, estas perspectivas se asientan en la concepción transformadora y empoderadora de la educación (Freire, 1993, 2001) y la comunicación (Kaplún, 1998). Se contribuye, así, a su conformación como sujetos políticos activos capaces de tomar decisiones de manera colectiva desde una perspectiva de derechos humanos (Martínez Guzmán y Ali, 2008). Para ello los cursos mencionados fomentan "la comprensión y la reflexión de las problemáticas, el reconocimiento de las diversas opiniones, creencias, percepciones o culturas y el desarrollo de una ciudadanía activa, es decir, la formación de ciudadanos empoderados que participen en el debate democrático" (Nos Aldás, Arévalo Salinas y Murphy, 2014, p. 116).

Por lo tanto, este tipo de docencia en comunicación no se centra únicamente en proponer alternativas a los modelos hegemónicos, sino en una eficacia comunicativa "alterativa" (Roncagliolo, 1988 en Marí Sáez, 2017). En efecto, se trata de transformar los marcos establecidos, desde sus raíces, problematizando y contextualizando las causas profundas de la violencia, la exclusión y la discriminación. Por lo tanto, la comunicación social pasaría a entenderse como el desarrollo de la política práctica (Hopgood, 2013) que tiene como finalidad "evaluar el contexto, enraizar los problemas de los sistemas imperantes y sugerir dónde pueden encontrarse alternativas progresistas [...] avanzar en un proyecto emancipador que tiene como objetivo profundizar en las condiciones para encontrar 
soluciones políticas de raíz" (Fenton, 2016, pp. 178-179)². Por otro lado, haciendo uso de una participación activa y equitativa en clase, este modelo de aprendizaje se detiene a repensar cómo las culturas están entrelazadas por procesos comunicativos complejos, por el lenguaje, los discursos, los procesos culturales y los movimientos sociales (Martín Barbero, 1998, 2014).

Al comprender la interdependencia de los actuales problemas y asumir una responsabilidad compartida a través de la comunicación, se establecen tres niveles diferentes de análisis tanto en la producción como en la evaluación de la comunicación. En primer lugar, anticipar y pensar en la idea (imagen) de (la) realidad representada. En segundo lugar, las relaciones que se conforman a través de dicha realidad. En tercer lugar, las reacciones que se buscan en la sociedad a través de esas representaciones: ¿se incita al odio o a la reconciliación? ¿a la inclusión o la exclusión? ¿al individualismo o al empoderamiento colectivo?

Con todo lo comentado anteriormente, el enfoque educativo propuesto se estructura desde una visión global y cosmopolita de los estudios mediáticos en sintonía con la educación para la ciudadanía crítica global enmarcada desde los derechos humanos (Starkey, 2017; Osler y Starkey, 2018). Se entiende que existen múltiples formas de vivir en el mundo y que existe una responsabilidad de comprenderlas y dialogar con ellas de forma colectiva (Zuckerman, 2013). Por ello, los procesos pedagógicos propuestos implican formar al estudiantado como sujetos políticos de una sociedad civil informada, comprometida y crítica (el principal "motor" para el cambio social), que participe de escenarios de comunicación orientados hacia una solidaridad global activa, además de interactuar a través de una información de calidad "comunicativa" (que incluya ilusión, esperanza y posibilidad) (Alfaro, 2005; DevReporter Network, 2016).

Sin embargo, los actuales contextos sociales, culturales y políticos han venido dificultando los procesos de aprendizaje reflexivos dentro de los contextos educativos formales, ya que requieren de tiempos más pausados. Recogiendo las aportaciones de Zygmunt (2005), la educación se enfrenta a una serie de retos dentro de la "modernidad líquida" donde la volatilidad y la incertidumbre constante dificultan el pensamiento crítico. En primer lugar, el autor (2005, p. 21) habla del "síndrome de la impaciencia”, ya que toda espera o dilación, también en los procesos de aprendizaje, supone un problema percibido, por lo que se opta por tomar atajos para conseguir lo deseado. En segundo lugar, el conocimiento se ha convertido en una adquisición, vinculado al consumismo, que sirve para ser usado una vez y sin valor de futuro: "Hoy el conocimiento es una mercancía; al menos se ha fundido en el molde de la mercancía y se incita a seguir formándose en concordancia con el modelo de mercancía” (Bauman, 2005, p. 30). En tercer lugar, la cantidad inabarcable de información recibida cada día, por multitud de canales, hace replantearse los fundamentos tradicionales de la educación, ya que en la actualidad el proceso de memorizar "produce más desperdicios que productos útiles” (Bauman, 2005, p. 37). Para afrontar estos procesos de cambio en el ámbito educativo, se necesitan metodologías participativas y de innovación educativa que nos aportan diferentes herramientas. Específicamente, en esta propuesta se trabaja el concepto de "sostenibilidad cultural" (Erro, 2010; Martín Barbero,

2 Traducción propia del original en inglés. 
2003), ya que apela a los tiempos necesarios para el aprendizaje, que deben impregnar, también, los tiempos de la comunicación transformadora.

Es por ello que el proceso de aprendizaje parte de los conocimientos y las experiencias vitales que el estudiantado posee al comienzo de la asignatura, construidos a través de procesos curriculares, culturales y sociales. De este modo, se aplican metodologías de historias de vida, con las que el estudiantado va construyendo de manera participativa y colectiva su recorrido educativo (Leite Méndez et al, 2016).

Se utilizan además herramientas y materiales de educación para una ciudadanía global para ir interpretando y relacionando sus propias experiencias, aprendizajes y marcos con las actitudes y valores de la justicia social. Por ejemplo, teniendo en cuenta el potencial educativo del juego como herramienta transformadora (Gil, Ramiro y Lozano, 2018) se utiliza una baraja de cartas de valores, elaborada por el Centro de Investigación de Interés Público (PIRC), basada en los valores de Schwartz con el objetivo de involucrar al estudiantado en diferentes problemáticas a nivel global. Por lo tanto, se parte de la teoría del encuadre (Darnton y Kirk, 2011) con el objetivo de transformar sus propias concepciones interiorizadas.

También es imprescindible abordar de manera crítica el papel que desempeñan el sensacionalismo y la política del consumo (Ouellette y Banet Weiser, 2018) en la construcción de pseudo-realidades. Casos recientes como la obtención de escaños por parte del partido de extrema derecha vox dentro del Parlamento andaluz, la elección de Bolsonaro como presidente de Brasil o el Brexit ponen sobre la mesa la necesidad de desarrollar propuestas pedagógicas críticas para cuestionar la influencia de los procesos comunicativos (a través de medios de comunicación tradicionales, redes sociales o la publicidad comercial) en la construcción de identidades y estilos de vida "egocéntricos" y "despolitizados".

En este sentido, uno de los puntos de partida para alcanzar los objetivos educativos propuestos, es tener en cuenta los diferentes modelos de socialización y aprendizaje del estudiantado a través de las plataformas digitales en las que se relacionan, y su influencia tanto en sus identidades colectivas como individuales. Así pues, se trata de asumir que los procesos de formación van más allá de los espacios educativos formales (Carpenter y McLuhan, 1974) dentro de contextos hiperconectados y globalizados, y que, en un proyecto de aprendizaje como esta asignatura, es preciso abordar tanto el potencial de los entornos digitales para desarrollar acciones activistas globales (Jenkins, 2016), como cuestionar su funcionamiento opaco y comercial.

\section{Implementación de experiencias docentes de grado y posgrado}

Partiendo del marco teórico y conceptual que se ha desarrollado anteriormente, en este apartado se presentan las prácticas pedagógicas concretas que se han implementado en dos programas de estudio, a nivel de grado y posgrado.

\subsection{Comunicación para la igualdad}

La asignatura de "Comunicación para la igualdad", de cuarto curso del plan de estudios del grado de Publicidad y Relaciones Públicas de la Universitat Jaume I, tiene carácter obligatorio y supone una mirada comunicativa y publicitaria más allá de las lógicas 
individualistas y comerciales para abordar también la perspectiva cultural y colectiva (Costa, 1995).

Es necesario que los futuros profesionales de la comunicación, incluida su vertiente publicitaria, puedan conocer las experiencias exitosas de las últimas tendencias en Comunicación para el Cambio Social. Por lo tanto, resulta vital el desarrollo de recursos y pedagogías docentes innovadoras para poder explicar teorías y prácticas sobre cómo transformar todo tipo de violencia desde la comunicación. Esto conlleva fortalecer los conocimientos del estudiantado sobre el rol de la violencia simbólica (Bourdieu y Passeron, 1996); de qué manera se legitiman y perpetúan, a través de los discursos, la violencia estructural y directa desde diferentes vertientes: violencia de género, pobreza, discursos del odio, entre otras. Así pues, un aprendizaje transformador pasa por implementar distintas estrategias comunicativas para desenmascarar las causas y estructuras que posibilitan estas situaciones desde sus inicios, desde la raíz, para revertirlas de manera noviolenta.

En esta asignatura transcendemos la mera descripción del sexismo en los medios y en la política, ya que es un enfoque necesario, pero no suficiente. Vinculamos el género a la erradicación de las desigualdades en el discurso y a la promoción de la justicia social y la cultura de paz, combinando una revisión epistemológica de las capacidades que posee la comunicación para activar sujetos políticos con metodologías innovadoras que desarrollan la conciencia y la implicación del estudiantado con estos temas (Gámez Fuentes, Nos Aldás y Farné, 2015, p. 36-37).

Para ello, en primer lugar, se posibilita la detección por parte del estudiantado de discursos (en medios de comunicación o campañas de publicidad) que fomentan y legitiman cualquier tipo de violencia e injusticia social (Penalva, 2002). A partir de aquí, a través de la creatividad comunicativa, se trabaja para analizar qué tipo de comunicación puede promover discursos pacíficos, justos y sin opresión. Es decir, se trata de reenmarcar las representaciones de la realidad (Lakoff, 2007; Darnton y Kirk, 2011) desafiando los marcos hegemónicos mediante nuevas narrativas (Reinsborough y Canning, 2017) que impulsen una comunicación innovadora y transformadora de las violencias, como las de género y que contemplen una perspectiva interseccional (Núñez Puente, Fernández Romero y Farné, 2018). Así, se estudian distintas acciones impulsadas por los movimientos sociales, campañas del Tercer Sector o guías con recomendaciones recogidas por el propio estudiantado, además de analizar cuáles han sido sus repercusiones sociales y culturales.

La realización de estos ejercicios previstos dentro del programa docente permiten al estudiantado no únicamente relacionarse con otras formas de comunicación transformadora que se han llevado a cabo de forma exitosa, sino, además, poner en duda todas aquellas nuevas tendencias comunicativas, basadas en la idea del respeto a las personas y al medioambiente, pero que responden a intereses de negocio y que no tienen una intencionalidad de cambio social (Gámez Fuentes y García López, 2015), como el pinkwashing o greenwashing.

Por lo tanto, a través de estos procesos de aprendizaje reflexivos, se trata de comprender que otros modelos de negocio son posibles, superando la idea de marca, la reputación y el enfoque comercial. De esta forma, se interpela al estudiantado para desafiar sus propios marcos profundos (Darnton y Kirk, 2011) a través de lo que Foss y Griffin (1995) definen como una retórica invitacional (que invite, que implique, sin imponer, sin una persuasión opaca), una comunicación dialógica (Kaplún, 1998) con el objetivo de construir una 
educación transformadora. Es decir, se parte de la idea de Canning y Reinsborough (2012) de que " $[\ldots .$.$] el obstáculo para convencer a la gente no suele ser lo que no saben, sino lo$ que ya saben. En otras palabras, son las conjeturas y creencias de las personas las que actúan como filtros narrativos que les impiden escuchar los mensajes de cambio social”s.

En primer lugar, se afrontan los límites de considerarse a sí mismos como consumidores individualistas y no como ciudadanos críticos y activos en la esfera mediática (Jenkins, 2006). Por esta razón, el modelo de aprendizaje que se utiliza enfrenta esta situación enseñando algunas ideas principales que ayudan a la conformación de una ciudadanía colectiva como "Lo personal es político" (Millet, 1970) y "Tu compra es tu voto" (Ballesteros, 2007), con el objetivo de abrir un abanico de posibilidades vinculadas a una economía social y solidaria y a proyectos políticos colectivos.

En segundo lugar, la permanente conexión con lo digital por parte del estudiantado conforma lo que Sampedro (2018, p. 48) denomina como quimeras de los nativos digitales, que se repiten de forma constante:

- Los nativos digitales tienen un dominio total de lo digital: asumen que poseen un conocimiento absoluto de los escenarios digitales, rechazando otras formas de conocimiento como la experiencia, la alfabetización mediática o el humanismo digital.

- Asumen que viven en una aldea global (digital): en determinadas ocasiones los más jóvenes se sienten conectados con el resto del mundo a través de Internet, ignorando que determinadas poblaciones no tienen acceso a las redes (brecha digital) o que los propios espacios digitales filtran la realidad.

- "Podemos ser quien queramos ser" dentro de un mundo conectado: el anonimato de las redes sociales y las comodidades del clic, construyen una idea de libertad exagerada, sin tener en cuenta los riesgos de la pérdida de privacidad (y la venta de información personal).

- "Las pantallas son suficientes": ya que "todo" se puede hacer en el entorno online, se desprecia lo que está "desconectado", ignorando la importancia de las relaciones y la actividad fuera de lo digital.

Por estas razones, una parte importante de la metodología del modelo educativo se construye a partir de lo que Sampedro (2018) ha denominado como "Dietética Digital". Es decir, incorporar una dieta saludable en el uso de las redes sociales a través de acciones en torno a la alfabetización mediática. Se trabaja con el estudiantado para identificar y entender las consecuencias políticas y pedagógicas del uso de las redes sociales sin ningún tipo de filtro.

Se trata, pues, de abrir espacios de reflexión en el aula para su conformación como sujetos políticos, como ciudadanía, identificando a los diferentes actores que conforman la cultura y que interactúan en diferentes entornos (online, offline y mediados) (Toret, 2013). De esta manera, esta asignatura tiene como objetivo general el desarrollo de las competencias del estudiantado y su agencia para que sean capaces de predecir las consecuencias culturales de la comunicación y realizar acciones comunicativas con incidencia política, que pasa por

s Traducción propia del original en inglés. 
trabajar en las diferentes áreas interconectadas de la vida social: un cambio social desde la comunicación, la educación, la legislación y la política.

Sin embargo, el reto se encuentra en llevar a la práctica un marco conceptual y teórico amplio a través de acciones pedagógicas concretas que necesariamente precisan de metodologías participativas y dialógicas para capacitar al estudiantado en el uso responsable de los escenarios digitales, pese a contar con 90 personas en las clases de teoría y 45 en cada una de las sesiones de prácticas. De este modo, tanto la teoría como la práctica se basan en procesos de aprendizajes cooperativos, reflexivos y participativos.

La asignatura se estructura en diferentes retos que posibilitan la implicación del estudiantado de manera progresiva, además de incrementar su motivación y compromiso. A continuación, se exponen:

\section{Introducción. Hackeando "Comunicación para la igualdad"}

El objetivo de las primeras clases es crear una conciencia grupal y conocer los conocimientos de partida del estudiantado. Para ello se llevan a cabo distintas actividades de activación y creación de grupo. Por otro lado, se trata de desactivar los prejuicios con los que los estudiantes llegan a la asignatura, ampliando el concepto de "igualdad" más allá de la aplicación de los estudios de género a la comunicación, por lo que se combina el enfoque del feminismo con el de la justicia social global interseccional y las culturas de paz.

\section{Reto 1: Comunicación publicitaria, diversidad, desacuerdo y cambio social.}

Se amplían los modelos de comunicación que han aprendido hasta el momento y se les dan a conocer otras alternativas comunicativas en el marco de la comunicación para el cambio social desde criterios colectivos, sociales y políticos. Además, se trabaja el concepto de "disidencia consensual" (Sampedro y Lobera, 2014) para que puedan ser capaces de implementar otras narrativas basadas en la no violencia y el diálogo.

\section{Reto 2: La eficacia cultural como nueva herramienta de trabajo}

Partiendo del concepto de "eficacia cultural" (Nos Aldás, 2007, 2010), se analizan críticamente estudios de caso vinculados a estrategias comunicativas de las ONGD. El objetivo es que se familiaricen con, y apliquen, criterios de comunicación propuestos por las investigaciones más recientes, los informes y documentos de trabajo de las principales organizaciones internacionales y las redes de comunicación para el cambio.

\section{Reto 3: Comunicación transgresora para el cambio social: aplicando las últimas tendencias}

A través de la exposición de prácticas comunicativas exitosas llevadas a cabo por diferentes organizaciones (por ejemplo, Fundación Quepo), el estudiantado conoce, comprende y produce otras formas de comunicar con intencionalidad de cambio social.

\section{Festival de eficacia cultural, conclusiones y revisión final de la experiencia de aprendizaje}

Después de los procesos de enseñanza comentados, el estudiantado realiza acciones comunicativas "transgresoras” y transformativas que son evaluadas en una sesión abierta al público por un jurado profesional, pero, también, por sus propios pares. En algunas ocasiones, bajo una metodología de aprendizaje-servicio, las campañas comunicativas son consensuadas y de utilidad para organizaciones reales. Las últimas sesiones de la materia están destinadas a revisar los procesos de aprendizaje, teniendo en cuenta que el 
profesorado ya ha evaluado las diferentes prácticas, por lo que es capaz de analizar y repasar con la clase los errores más representativos, así como posibles mejoras.

A través de este diseño curricular basado en retos, se aplica una metodología de aprendizaje continua y participativa, respetando el propio ritmo del estudiantado:

- El estudiantado trabaja en grupos para colaborar con el profesorado en dinamizar las sesiones, otorgándole un papel relevante para que decidan cuáles son las mejores maneras de aprender de forma cooperativa entre iguales, diseñando las propias dinámicas de clases (supone un 10\% de la nota final).

- Realizan un mapa conceptual final: sintetizan y plasman lo aprendido a través de metodologías de pensamiento creativo y visual ( $20 \%$ de la nota).

- Proyecto final (vinculado al Festival de Eficacia Cultural): se trata de desarrollar una acción comunicativa en la que apliquen los conceptos teóricos aprendidos (30\% de la nota), como "eficacia cultural" o comunicación "transgresora".

- Elaboran un portfolio de aprendizaje como un diario de aprendizaje, donde desarrollan habilidades de reflexión y claridad expresiva, al tiempo que demuestran la comprensión de los nuevos contenidos. En este espacio recogen los conceptos más importantes, los debates del aula y las buenas prácticas comunicativas detectadas ( $40 \%$ de la nota).

En conclusión, se invita al estudiantado a aplicar de manera constante lo aprendido durante el curso y a "que tomen en cuenta su cultura, que sean independientes, interactivos y coherentes con los objetivos de aprendizaje” (UNESCO, 2015, p. 54). Es por ello que la elaboración de narrativas transgresoras permite abordar temas controvertidos de forma reflexiva (Oxfam, 2018) con el objetivo de entender la multitud de posibilidades de actuación con intención transformadora desde el espacio educativo y comunicacional.

\subsection{Comunicación para la paz}

La otra experiencia docente vinculada a promover la educación en justicia global es la asignatura "Comunicación para la paz" del Máster Universitario en Estudios Internacionales de Paz, Conflictos y Desarrollo de la Universitat Jaume I de Castellón. Este postgrado tiene una trayectoria de casi veinticinco años y desde el curso 2006-2008 se ha adaptado al Espacio Europeo de Educación Superior (EEES), convirtiéndose en un programa oficial de 120 ECTS y con una duración de dos años. Para entender mejor la asignatura, es necesario mencionar algunas características específicas de este Máster, especialmente su faceta internacional, intercultural e interdisciplinar (Farné, 2017, 2019).

Ante todo, el estudiantado proviene de diferentes países de todos los continentes, por lo que no solo conforma una gran interculturalidad, sino que está acostumbrado a sistemas y enfoques educativos muy diferentes. En segundo lugar, es un programa interdisciplinar vinculado a la Facultad de Ciencias Humanas y Sociales y sus estudiantes provienen de una variedad de estudios de grado (como Filosofía, Historia, Filología, Educación e incluso de varias Ingenierías o Medicina) por lo que están familiarizados con diferentes estilos y lenguajes académicos, dependiendo de su campo previo. Un tercer punto a tener en cuenta es que este Máster ofrece cursar los estudios en dos idiomas, español o inglés, pero la mayoría de estudiantes no son nativos de estos idiomas. Aunque para matricularse necesiten un nivel B2 o superior (que, según el marco europeo de lenguas, corresponde a un intermedio alto), en muchos casos el idioma supone una complejidad añadida. Estos 
tres factores componen un grupo de estudiantes con intereses y necesidades muy diferentes y que se tienen que tomar en cuenta al diseñar temarios y metodologías adecuadas para esta idiosincrasia.

En este contexto, la asignatura "Comunicación para la paz” se imparte en español e inglés (“Communication for peace”) en el primer semestre del primer año del Máster y sigue la estructura de otras asignaturas del programa. Es un curso intensivo de cuatro semanas con tres semanas de clases (3 horas al día) y una cuarta semana de investigación no presencial para que cada estudiante escriba su trabajo de curso. Siguiendo el enfoque del EEES centrado en el proceso de aprendizaje de cada estudiante, los 6 créditos ECTS se distribuyen en 45 horas de clases presenciales (30\% del total) y 105 horas de trabajo personal por parte de cada estudiante (70\%) que incluye tanto las horas de preparación de actividades durante las primeras tres semanas lectivas como las horas para investigar y volcar el aprendizaje en el trabajo escrito del curso.

Con relación a la temática, "Comunicación para la paz" aborda el rol de la comunicación para promover culturas de paz, igualdad y justicia social desde el análisis de la violencia simbólica y cultural y la creación de relatos de culturas de paz. Dado que la mayoría de estudiantes de este Máster no proviene de grados del área de comunicación, el enfoque de la asignatura está adaptado pensando en el perfil interdisciplinar del estudiantado en lugar de ser una formación específica para perfiles de comunicación. Con ello se persigue que los futuros profesionales de la cooperación, o de los diferentes sectores donde puedan aplicar los aprendizajes sobre paz, conflictos y desarrollo, tomen conciencia del rol de la comunicación en la construcción de discursos que promuevan la transformación de injusticias y desigualdades y que así puedan incorporar una mirada comunicativa a sus diferentes ámbitos de trabajo. Para ello, “el curso está diseñado desde un concepto amplio de comunicación con el objetivo de analizar, discutir y comprender cómo los discursos se han diseñado a lo largo de la historia y cómo la forma en que la realidad se construye en los medios de comunicación masivos y en otros espacios de comunicación influyen en nuestros comportamientos y nuestras relaciones sociales y culturales” (Farné y López Ferrández, 2016, p. 653).

En este sentido, la asignatura trabaja de manera transversal diferentes elementos de la sostenibilidad, especialmente en su vertiente sociocultural, poniendo de relieve las interrelaciones del actual sistema y explorando posibles alternativas, centrándose en cómo estas se pueden trabajar comunicativamente. Desde un punto de vista teórico-conceptual, la vinculación de esta asignatura con los oDs se basa principalmente en los enfoques de Comunicación para el Cambio Social (Tufte, 2015), que recogen las tradiciones de Comunicación y Educación para el Desarrollo (FAO, 2007; Mesa, 2010). Concretamente, estos enfoques se vinculan también con los Estudios Culturales, de Paz y Feministas (Gámez Fuentes, Nos Aldás y Farné, 2015), además de incluir una perspectiva activista para que los conocimientos adquiridos se plasmen en una capacidad para transformar el sistema de injusticias y desigualdades (Comins y Muñoz, 2013). Además, están enfocadas a incorporar estos temas especialmente desde el punto de vista de futuros profesionales de la comunicación; que tengan muy presentes en sus proyectos y campañas elementos de sostenibilidad social, medioambiental, económica y cultural (Nos Aldás, 2007; Tucker, 2015).

A partir del cruce entre las perspectivas teórico-pedagógicas y los contenidos del plan de estudio, hemos elaborado un temario estructurado en los siguientes bloques: 
- Introducción a la comunicación y a las teorías mediáticas. Esta primera fase se centra en dos aspectos: 1) revisión y discusión de conceptos básicos en ciencias de la comunicación y principales enfoques teóricos y 2) reflexión sobre los marcos comunicativos y sus consecuencias en la construcción de discursos.

- Comunicación e investigación para la paz: 1) marco teórico y metodológico de comunicación para la paz y 2) comunicación para la paz como incidencia política y comunicación del cambio social.

- Acción comunicativa social y estrategias de storytelling: herramientas de análisis del poder narrativo (localizar nuevos marcos, reconocer el rol de las emociones y transformación de escenarios comunicativos).

- Análisis, discusión, planificación y evaluación de buenas prácticas comunicativas de comunicación de cambio social: 1) Comunicación activista de cambio social y 2) comunicación y divulgación (campañas y trabajo académico para el cambio social).

- Conclusiones: puesta en común, revisión colectiva y auto-evaluación de los aprendizajes.

Siguiendo estos enfoques y objetivos, adoptamos una metodología donde todos y todas aprendemos del resto, tanto estudiantado como profesorado. El proceso de enseñanza está centrado en el estudiantado, en sus conocimientos previos y en un aprendizaje autónomo. Las clases son una combinación de explicaciones, dinámicas participativas y trabajo práctico, con el apoyo de diferentes materiales (lecturas de artículos y libros, recursos audiovisuales, páginas web y redes sociales de instituciones, organizaciones y movimientos relevantes en el ámbito de la comunicación para la paz y el cambio social). Esta metodología se puede aplicar en un contexto de Máster con clases de máximo 25 estudiantes, facilitando así un aprendizaje interactivo.

De esta manera, se persigue que el estudiantado adquiera distintas competencias para ser capaz de "juzgar sus informaciones, sus textos y sus discursos y poder valorarlos de una manera autónoma y creativa; promover el diálogo de forma abierta e inclusiva teniendo en cuenta los presupuestos de estos estudios; utilizar de modo claro y sin ambigüedades la terminología propia en este ámbito interdisciplinar; y comunicar de forma escrita, mediante la realización de trabajos académicos, los contenidos aprendidos en los estudios de paz, conflictos y desarrollo" (Farné y López Ferrández, 2016, p. 654).

Estos procesos de enseñanza activan también se apoyan en una evaluación formativa y continua del estudiantado. Concretamente, la evaluación contempla tres tareas.

- Asistencia y participación en clase (20\% de la nota final): se promueve, entrena y evalúa la participación activa, con la preparación previa de lecturas y materiales, las aportaciones analíticas y críticas a los debates, así como la dinamización de algunos materiales complementarios con fines pedagógicos para el conjunto de la clase.

- Presentación oral (20\% de la nota final): se promueve el trabajo cooperativo para que el estudiantado trabaje en grupos pequeños (habitualmente entre 3 y 5 personas) para preparar una campaña de comunicación para el cambio social. En la última semana del curso, se organiza una sesión en formato de festival para la 
presentación de todas las campañas y el estudiantado participa en asignar un premio a la mejor campaña a través de la evaluación por pares.

- Un trabajo académico escrito (60\% de la nota final): consiste en un trabajo individual sobre una temática vinculada a comunicación para la paz y el cambio social de libre elección por cada estudiante, a elegir entre el formato de ensayo académico (con un estudio de caso sobre un producto comunicativo) o un proyecto práctico (se realiza una campaña de comunicación para la paz, diferente a la presentada en grupo). Para facilitar el aprendizaje en la elaboración de estos trabajos, se ha introducido una experiencia de innovación docente con un taller teórico-práctico en los últimos días de clase para que el estudiantado incorpore aprendizajes específicos en la redacción de trabajos académicos a través de revisión por pares (Farné, 2019).

\section{Conclusiones}

El desarrollo de una educación para la ciudadanía crítica global, en relación con los Objetivos de Desarrollo Sostenible, requiere la construcción de nuevas metodologías docentes que permitan al estudiantado comprender de forma pausada y reflexiva las distintas caras e interconexiones de múltiples realidades. Es por ello que la implementación de las dos asignaturas presentadas a lo largo de la investigación ha supuesto desarrollar nuevos procesos pedagógicos vinculados a la docencia en comunicación dentro del ámbito universitario. Los temas que se tratan, así como las actividades propuestas, están diseñados para que el estudiantado pueda tener a su disposición un conjunto de herramientas teórico-prácticas con las que analizar y realizar acciones comunicativas con vocación de transformación social. Esto supone asumir que el profesorado, a diferencia de las concepciones clásicas educativas, es un agente facilitador para la construcción de espacios de aprendizaje activo, donde el estudiantado sea capaz de desarrollar sus propias competencias como ciudadanía crítica.

El diseño de las asignaturas "Comunicación para la igualdad” y "Comunicación para la paz” se basa en un proceso de evaluación continua del estudiantado, con el objetivo de mejorar los procesos de aprendizaje progresivo. Ambos programas tienen en cuenta un proceso de evaluación holística que se compone de distintas fases: diagnóstica, formativa y sumativa (Vallés, Ureña y Ruiz, 2011). Por otro lado, la implicación del estudiantado, por ejemplo, en la evaluación por pares, entre iguales, supone la introducción de aspectos democráticos, reflexivos y recíprocos en consonancia con los valores de aprendizaje. Además, es importante mencionar que se tienen en cuenta las valoraciones anuales del estudiantado para incorporar nuevos aspectos en las metodologías docentes.

Sus reflexiones y propuestas aluden siempre al doble proceso personal y profesional que desarrollan durante las asignaturas, ya que observan una transformación de su mirada crítica como personas, pero, también, de su capacitación cultural como comunicadores y comunicadoras. Además, valoran muy positivamente la posibilidad de desarrollar una campaña real con valores sociales y exponerla en un Festival de Eficacia Cultural como cierre de curso frente a profesionales del sector (en el caso de la asignatura de Grado). Por otro lado, la experiencia de dinamizar una sesión de clase es otro de los aspectos que consideran enriquecedor en su formación, al crearse espacios de discusión y debate entre sensibilidades muy diferentes y poder participar de la toma de decisiones relacionadas con su aprendizaje. 
En conclusión, estas propuestas educativas parten de nuestros resultados de investigación desde enfoques iterativos (Seguí Cosme y Nos Aldás, 2017) en los que el debate entre teoría y práctica, así como la transferencia entre los diferentes agentes de la Comunicación del Cambio Social (incluidas profesionales de las organizaciones y activistas) permiten la actualización constante de herramientas que posibiliten una transformación social de forma colectiva. Entendemos, por tanto, que el desarrollo de una enseñanza superior basada en los valores de la educación para la ciudadanía crítica global requiere incorporar criterios de comunicación movilizadores (y transgresores) dentro de los procesos pedagógicos. De esta manera, no solo se posibilita la conformación de un estudiantado activo en contextos educativos formales, sino que, además, se establecen las condiciones necesarias para constituirse como sujetos políticos (como ciudadanos activos) capaces de tomar decisiones de manera reflexiva, crítica y autónoma bajo criterios de justicia social.

\section{Agradecimientos}

Resultados del Seminario Permanente de Innovación Educativa (SPIE 3608) COMCAMBIO "Menú digital saludable para el aprendizaje crítico-participativo" (Convocatoria de ayudas para proyectos de innovación educativa USE-UJI) y de los proyectos MINECO CSO2013-48612-C2-1-P "Prácticas y perfiles tecnopolíticos. Nociones emergentes de ciudadanía” y FEM2015-65834-C2-2-P "La resignificación de la mujer-víctima en la cultura popular: implicaciones para la innovación representacional en la construcción de la vulnerabilidad y la resistencia". También ha sido posible su realización gracias a las Ayudas para la formación de profesorado universitario (FPU 17/02431) del Ministerio de Ciencia, Innovación y Universidades.

\section{Referencias}

Alfaro, R. M. (2005). Sociedades en movimiento: Desafíos comunicativos a la sociedad de la información. En J. Echevarría (Ed.), Solidaridad en red. Nuevas tecnologías, ciudadanía y cambio social (pp. 97-125) Bilbao: Hegoa.

Arévalo Salinas, A. I., Vilar Sastre, G. y Al-Najjar Trujillo, T. (2018). Periodismo de paz y medios alternativos digitales en España. En A. I. Arévalo Salinas, G. Vilar Sastre y AT. 1-Najjar Trujillo (Eds.), Comunicación, paz y conflictos (pp. 17-28). Madrid: Dykinson. https://doi.org/10.2307/j.ctv9hvtdx.4

Austin, J. L. (1976). How to do things with words. Oxford: Oxford University Press. https://doi.org/10.1093/acprof:oso/9780198245537.001.0001

Ballesteros, C. (2007). Tu compra es tu voto: consumo responsable, ecología y solidaridad. Madrid: HOAC.

Bourdieu, P. y Passeron, J. C. (1996). La reproducción. Elementos para una teoría del sistema de enseñanza. Ciudad de México: Fontamara.

Bourn, D. (2018). Global citizenship education: A critical introduction to key concepts and debates. Policy E Practice: A Development Education Review, 27, 199-203.

Bugallo Rodríguez, A. y Naya Riveiro, M. C. (2018). Educación para la ciudadanía global (ECG): Comprendiendo lo internacional a través de lo local. Revista Lusófona De Educação, 41, 139151. https://doi.org/10.24140/issn.1645-7250.rle41.09

Canning, D. y Reinsborough, P. (2012). Narrative power analysis. En A. Boyd y D. O. Mitchell (Eds.), Beautiful trouble. A toolbox for revolution. Londres: OR Books. 
Carpenter, E. y McLuhan, M. (1974). El aula sin muros: Investigaciones sobre técnicas de comunicación. Barcelona: Icaria.

Comins, M. I. y Muñoz, F. A. (2013). Filosofía y praxis de la paz. Barcelona: Icaria.

Costa, J. (1995). Crisis y crítica de la publicidad El Narciso que se cree Jano. Telos: Cuadernos de Comunicación, Tecnología y Sociedad, 42, 28-34.

Crenshaw, K. (1991). Mapping the margins: Intersectionality, identity politics, and violence against women of color. Stanford Law Review, 43, 1241-1299. https://doi.org/10.2307/1229039

Darnton, A. y Kirk, M. (2011). Finding frames: New ways to engage the UK public in global poverty. Londres: OXFAM-Bond for International Development.

DevReporter Network. (2016). Vademécum: Principios para una información internacional responsable. Recuperado de http://devreporternetwork.eu/es/le-vademecum-principes-pour-unemeilleure-information-sur-la-solidarite-internationale-et-les-pays-du-sud/

Duncombe, S. (2007). Dream: Re-imagining progressive politics in an age offantasy. Nueva York, NY: The New Press.

FAO. (2007). Comunicacioón y desarrollo sostenible. Roma: Organizaciones de las Naciones Unidas para la Agricultura y la Alimentación.

Farné, A. (2017). Approaching education practices of social change: A post-graduate learning experience in an intercultural setting. En L. Gómez Chova, A. López Martínez e I. Candel Torres (Eds.), Proceedings of 10th International Conference of Education, Research and Innovation (pp. 5288-5293). Sevilla: Universidad de Sevilla. https://doi.org/10.21125/iceri.2017.1386

Farné, A. (2019). Enhancing students' academic writing skills at post-graduate level: An active learning experience in an intercultural context. En INTED (Ed), Proceedings of 13th annual International Technology, Education and Development Conference (pp. 6068-6073). Valencia: IATED. https://doi.org/10.21125/inted.2019.1483

Farné, A. y López Ferrández, F. J. (2016). Comunicación para la paz: Una propuesta pedagógica para el cambio social. Opción. Revista de Ciencias Humanas y Sociales, 32(9), 644-662.

Farné, A., Castillo Mateu, L. y Nos Aldás, E. (mayo, 2018). Communication towards equality for future media professionals: Implementing social awareness through a cultural efficacy festival. Comunicación presentada en el International Conference 'Trial and Error'. Universidade do Minho.

Fenton, N. (2016). Digital, political, radical. Malden: Polity Press.

Foss, S. K. y Griffin, C. L. (1995). Beyond persuasion: A proposal for an invitational rhetoric. Communication Monographs, 62, 2-18. https://doi.org/10.1080/03637759509376345

Freire, P. (1993). Pedagogía de la esperanza: Un reencuentro con la pedagogía del oprimido. Madrid: Siglo XXI.

Freire, P. (2001). Pedagogía de la indignación. Madrid: Morata.

Galtung, J. (1996). Peace by peaceful means: Peace and conflict. Development and civilization. Oslo: SAGE.

Gámez Fuentes, M. J. y García López, M. (2015). Las marcas y la seducción del activismo. En E. Nos Aldás, A. Arévalo Salinas y A. Farné (Eds.), Comunicación y sociedad civil para el cambio social (pp. 426-437). Madrid: Fragua.

Gámez Fuentes, M. J., Nos Aldás, E. y Farné, A. (2015). Communication towards equality in the european higher education area: Building capacities for social change with Spanish 
undergraduates. The International Journal of Learning in Higher Education, 22(3), 33-44. https://doi.org/10.18848/2327-7955/CGP/v22io3/48621

Gil, J. V., Ramiro, E. y Lozano, N. (2018). El juego como estrategia para la transformación de conflictos desde la educación. Una propuesta para educar en la interculturalidad. En A. I. Arévalo Salinas, G. Vilar Sastre y T. Al-Najjar Trujillo (Eds.), Comunicación, paz y conflictos (pp. 51-59). Madrid: Dykinson. https://doi.org/10.2307/j.ctv9hvtdx.7

Giroux, H. y Mclaren, P. (1993). Between borders: Pedagogy and the politics of cultural studies. Nueva York, NY: Routledge.

Hall, S. (1997). Representation: Cultural representations and signifying practices. Londres: SAGE.

Herrero, Y. (2013). Miradas ecofeministas para transitar a un mundo justo y sostenible. Revista de Economía Crítica, 16, 278-307.

Hooks, B. (1994). Teaching to transgress. Education as the practice of freedom. Londres: Routledge.

Hopgood, S. (2013). The endtimes of human rights. Nueva York, NY: Cornell University Press.

Jenkins, H. (2006). Fans, bloggers, and gamers: Exploring participatory culture. Nueva York, NY: New York University Press.

Jenkins, H. (2016). By any means necessary. The new youth activism. Nueva York, NY: New York University Press.

Kaplún, M. (1998). Una pedagogía de la comunicación. Madrid: La Torre.

Lagarde y de los Ríos, M. (2005). Para mis socias de la vida. Claves feministas. Madrid: Editorial horas y horas.

Lakoff, G. (2004). Don't think of an elephant: Know your values and frame the debate. Montpelier, VE: Chelsea Green.

Laville, J. L. y Jané, J. G. (2009). Crisis capitalista y economía solidaria. Una economía que emerge como economía real. Barcelona: Icaria Antrazyt.

Leite Méndez, A., Cortés González, P. y Rivas Flores, J. I. (2016). Narrativa y creatividad en la universidad: ¿Es posible transitar otros caminos en la enseñanza y el aprendizaje? En P. Cortés González y M. J. Marqués García (Eds.), Creatividad, comunicación y educación. Más allá de las fronteras del saber establecido (pp. 151-164). Málaga: Universidad de Málaga.

López Ferrández, F. J. (2016). El colectivo enmedio y su campaña fiesta cierra-bankia: Experiencias de comunicación para el cambio social a través del espectáculo ético. Revista Científica de Información y Comunicación, 13, 87-115.

Marí Sáez, V. (2017). Towards a critical political economy of indicators. Measuring and evaluating "alteratively" communication, development and social change. Commons. Revista de Comunicación y Ciudadanía Digital, 6(1), art 1. https://doi.org/10.25267/COMMONS.2017.v6.i1.01

Martín Barbero, J. (1998). Heredando el futuro: Pensar la educación desde la comunicación. Cultura y Educación, 9, 17-36. https://doi.org/10.1174/113564098760604947

Martín Barbero, J. (2003). La educación desde la comunicación. Bogotá: Norma.

Martín Barbero, J. (2014). Jesús Martín Barbero: Conceptos clave en su obra. Recuperado de https://www.youtube.com/watch?v=NveV5ScaZHg

Martínez Guzmán, V. (2001). Filosofía para hacer las paces. Barcelona: Icaria.

Martínez Guzmán, V. (2005). Podemos hacer las paces. Bilbao: Desclée de Brouwer. 
Martínez Guzmán, V. y Ali, F. A. (2008). Education for human right to peace from a perspective of philosophy for making peace(s). Cuadernos Constitucionales de la Cátedra Fadrique Furió Ceriol, 62, 197-201.

Mesa, M. (2010). Comunicación y educación para el desarrollo: Una apuesta por la ciudadanía global. En J. Erro Sala y T. Burgui (Eds.), Comunicando para la solidaridad y la cooperación. cómo salir de la encrucijada (pp. 201-220). Pamplona: Foro de Comunicación, Educación y Ciudadanía.

Mesa, M., Alonso Cano, L. y Couceiro, E. (2013). Visibles y transgresoras. Narrativas y propuestas visuales para la paz y la igualdad. Madrid: CEIPAZ.

Mies, M. y Shiva, V. (1993). Ecofeminism. Londres: Zed Books.

Millet, K. (1970). Sexual politics. Nueva York, NY: Doubleday \& Company.

Naciones Unidas. (1999). Declaración y programa de acción sobre una cultura de paz. Recuperado de http://undocs.org/es/A/RES/53/243.

Naciones Unidas. (2015). Objetivos y metas de desarrollo sostenible. Recuperado de https://www.un.org/sustainabledevelopment/es/sustainable-development-goals/

Nos Aldás, E. (2007). Lenguaje publicitario y discursos solidarios: Eficacia publicitaria, ¿̨eficacia cultural? Barcelona: Icaria.

Nos Aldás, E. (2010). Comunicación, cultura y educación para la solidaridad y el desarrollo. En J. Erro Sala y T. Burgui (Eds.), Comunicando para la solidaridad y la cooperación. Cómo salir de la encrucijada (pp. 113-135). Pamplona: Foro de Comunicación, Educación y Ciudadanía.

Nos Aldás, E. (2019). Learning with 'generation like' about digital global citizenship: A case study from Spain. En D. Bourn (Ed.), Bloomsbury handbook for global education and learning (pp. 145167). Londres: Bloomsbury.

Nos Aldás, E., Arévalo Salinas, A. I. y Murphy, J. (2014). Acción social comunicativa desde la universidad. En M. García López (Coord.), El papel de la universidad en los procesos de comunicación y cooperación para el desarrollo social y humano (pp. 113-132). Madrid: Fragua.

Núñez Puente, S., Fernández Romero, D. y Farné, A. (2018). Comunicación, violencia de género y prácticas de resistencia: Narrativas innovadoras para un cambio social. Teknokultura, 15(2), 185-192. https://doi.org/10.5209/TEKN.61602

Osler, A. y Starkey, H. W. (2018). Extending the theory and practice of education for cosmopolitan citizenship. Educational Review, $70(1)$, 31-40. https://doi.org/10.1080/00131911.2018.1388616

Ouellette, L. y Banet Weiser, S. (2018). Media and the extreme right. Communication, Culture and Critique, 11(1), 1-6. https://doi.org/10.1093/ccc/tcx02 1

Oxfam. (2007). Escuelas y educación para la ciudadanía global. Una mirada transformadora. Barcelona: Saberes.

Oxfam. (2018). Teaching controversial issues. A guide for teachers. Recuperado de https://www.oxfam.org.uk/education/resources/teaching-controversial-issues.

Penalva, C. (2002). El tratamiento de la violencia en los medios de comunicación. Alternativas. Cuadernos de Trabajo Social, 10, 395-412. https://doi.org/10.14198/ALTERN2002.10.31

Pinazo, D. y Nos Aldás, E. (2016). Developing moral sensitivity through protest scenarios in international NGDOS communication. Communication Research, 43(1), 25-48. https://doi.org/10.1177/0093650213490721

Ramonet, I. (1998). La tiranía de la comunicación. Madrid: Debate. 
Reinsborough, P. y Canning, D. (2017). Re-imagining change. Oakland: PM Press.

Roncagliolo, R. (1988). Las redes de cooperación y la radio comunitaria. En M. Chaparro (Ed.), La democratización de los medios (pp. 59-78). Sevilla: Diputación de Sevilla.

Sampedro, V. (2018). Dietética digital. Para adelgazar al gran hermano. Barcelona: Icaria.

Sampedro, V. y Lobera, J. (2014). The Spanish 15-M movement? Journal of Spanish Cultural Studies, 15, 61-80. https://doi.org/10.1080/14636204.2014.938466.

Seguí Cosme, S. y Nos Aldás, E. (2017). Bases epistemológicas y metodológicas para definir indicadores de eficacia cultural en la comunicación del cambio social. Commons. Revista de Comunicación y Ciudadanía Digital, 6(2), 10-33.

https://doi.org/10.25267/COMMONS.2017.v6.i2.02

Sen, A. (1999). Development as freedom. Oxford: Oxford University Press.

Starkey, H. W. (2017). Globalization and education for cosmopolitan citizenship. En J. A. Banks (Ed.), Citizenship education and global migration: Implications for theory, research, and teaching. Washington, DC: American Educational Research Association.

Toret, J. (2013) Technopolitics: the power of connected multitudes. 15M network-system and the new paradigm of distributed politics. Recuperado de http://datanalysis 15m.files.wordpress.com/2013/06/technopolitics-slides.pdf

Torres, C. A. (2017). Theoretical and empirical perspectives of critical global citizenship education. Londres: Routledge. https://doi.org/10.4324/9781315452579

Tucker, W. (2015). How to communicate the sustainable development goals to the public. Recuperado de https://www.theguardian.com/global-development-professionalsnetwork/2015/sep/07/how-to-communicate-the-sustainable-development-goals-to-thepublic

Tufte, T. (2015). Comunicación para el cambio social. La participación y el empoderamiento como base para el desarrollo mundial. Barcelona: Icaria.

Tufte, T. (2017). Communication and social change: A citizen perspective. Newark, NJ: Wiley.

Tuvilla Rayo, J. (2004). Cultura de paz y educación. En F. A. Muñoz (Ed.), Manual de paz y conflictos (pp. 390-425). Granada: Universidad de Granada.

UNESCO. (2012). Educación para el desarrollo sostenible. París: UNESCO.

UNESCO. (2015). Educación para la ciudadanía mundial. Temas y objetivos de aprendizaje. París: UNESCO.

Vallés, C., Ureña, N. y Ruiz, E. (2011). La evaluación formativa en docencia universitaria. Resultados globales de 41 estudios de caso. REDU. Revista de Docencia Universitaria, 9(1), 22-32. https://doi.org/10.4995/redu.2011.6184

Zuckerman, E. (2013). Rewire: Digital cosmopolitans in the age of connection. Nueva York, NY: WW Norton \& Co.

Zygmunt, B. (2005). Los retos de la educación en la modernidad líquida. Barcelona: Gedisa.

\section{Breve CV de los autores}

\section{Eloísa Nos Aldás}

Catedrática de la Universitat Jaume I de Castellón (UJI) en el Departamento de Ciencias de la Comunicación. Tiene una trayectoria investigadora, docente y de gestión de 21 años 
sobre Comunicación, Sociedad Civil y Cambio Social. Imparte clases de "Comunicación para la Igualdad" en el Grado de Publicidad y RRPP y de "Comunicación para la Paz" en el Máster Universitario en Estudios Internacionales de Paz, Conflictos y Desarrollo. Desde 2010 a 2016 fue directora de la sede-UJI del Instituto Interuniversitario de Desarrollo Social y Paz (IUDESP), en el que continua como investigadora. Ha realizado estancias internacionales, entre otras, en el desaparecido Teachers College de la Columbia University de Nueva York y el Development Education Research Center (DERC) de la UCL de Londres. ORCID ID: https://orcid.org/0000-0002-8871-976X. Email: aldas@uji.es

\section{Alessandra Farné}

Investigadora postdoctoral del Dpto. de Ciencias de la Comunicación de la Universitat Jaume I y miembro del grupo de investigación e Instituto Interuniversitario de Desarrollo Social y Paz. Licenciada en Relaciones Públicas con un Máster y Doctorado en Estudios Internacionales de Paz, Conflictos y Desarrollo. Líneas de investigación: comunicación para la paz y el cambio social, comunicación de la sociedad civil y (ciber)activismo. La actividad docente de Grado y Máster también está vinculada a los temas de comunicación para la paz, la igualdad y cooperación al desarrollo. Participación en varios proyectos de I + D sobre comunicación para para el cambio social y en actividades de transferencia del conocimiento con organizaciones de la sociedad civil. ORCID ID: https://orcid.org/0000-0002-5431-9143. Email: farne@uji.es

\section{Tamer Al-Najjar Trujillo}

Personal investigador en formación predoctoral (FPU17/02431) adscrito al Departamento de Ciencias de la Comunicación y el Instituto Interuniversitario de Desarrollo Social y Paz de la Universitat Jaume I. Graduado en Periodismo por la Universidad Carlos III de Madrid y Máster en Estudios Internacionales de Paz, Conflictos y Desarrollo por la UJI. Línea de investigación: el papel de la Comunicación y el Periodismo para la $\mathrm{Paz}$ en la prensa digital alternativa española. ORCID ID: https://orcid.org/0000-0002-4113-4502.Email: alnajjar@uji.es 